\title{
Cell-Based Therapies for Diabetic Complications
}

\author{
Stella Bernardi, ${ }^{1,2}$ Giovanni Maria Severini, ${ }^{3}$ Giorgio Zauli, ${ }^{3}$ and Paola Secchiero ${ }^{1}$ \\ ${ }^{1}$ Department of Morphology and Embriology and LTTA Centre, University of Ferrara, 44100 Ferrara, Italy \\ ${ }^{2}$ Baker IDI, Heart \& Diabetes Institute, Melbourne, VIC 3004, Australia \\ ${ }^{3}$ Institute for Maternal and Child Health, IRCCS Burlo Garofolo, Trieste, 34137, Italy \\ Correspondence should be addressed to Paola Secchiero, paola.secchiero@unife.it
}

Received 24 February 2011; Accepted 21 March 2011

Academic Editor: Gian Paolo Fadini

Copyright (c) 2012 Stella Bernardi et al. This is an open access article distributed under the Creative Commons Attribution License, which permits unrestricted use, distribution, and reproduction in any medium, provided the original work is properly cited.

In recent years, accumulating experimental evidence supports the notion that diabetic patients may greatly benefit from cell-based therapies, which include the use of adult stem and/or progenitor cells. In particular, mesenchymal stem cells and the circulating pool of endothelial progenitor cells have so far been the most studied populations of cells proposed for the treatment of vascular complications affecting diabetic patients. We review the evidence supporting their use in this setting, the therapeutic benefits that these cells have shown so far as well as the challenges that cell-based therapies in diabetic complications put out.

\section{Introduction}

The worldwide increase in the prevalence of diabetes mellitus reinforces the search for solutions to prevent it as well as to oppose the development and the progression of its complications. Particularly, the increasing prevalence of diabetes mellitus (DM) now affects adolescents and younger adults, thus promoting an earlier development of invalidating chronic diseases [1]. Experimental evidence suggests that cell-based therapies might represent a new and promising strategy for the treatment of diabetic vascular complications, and growing interest has recently been focused on mesenchymal stem cells and endothelial progenitor cells. Both cells types not only act against the mechanisms underlying diabetic complications but also rescue the abnormalities that stem cells present in diabetic patients, which contribute to the vascular complications. Notably, these cells avoid the ethical issues relating to the use of the embryonic cells. However, there are concerns about how the diabetic environment affects these cells. So, additional challenges for these cells include making them resistant to the diabetic environment and thus increasing their clinical efficacy [2].

On these premises, we will here review the evidence suggesting why adult stem/progenitor cells should be used in diabetic patients, the therapeutic benefits that these cells seem to offer for treating macrovascular and microvascular complications, and the challenges that cell-based therapies in DM present.

\section{Stem Cells}

Adult stem cells comprise of roughly 3 different groups: the bone marrow stem cells (BM-SC), the circulating pool of stem/progenitor cells (which are also derived from the bone marrow), and the tissue-resident stem cells. BM-SC can be further categorized into multipotent adult progenitor cells, mesenchymal stem cells (MSC), and hematopoietic stem cells. The circulating pool of stem/progenitor cells includes different types of cells, among which the most studied for the setting of vascular complications are the endothelial progenitor cells (EPC). EPC were identified by Asahara et al. [3] in the search for circulating angiogenic cells. They observed that these cells were able to form new blood vessels and promote neovascularisation after ischemia. Therefore, these cells seem to be the most promising in the setting of DM because of their potential utility in therapeutic neovascularisation and vascular repair. This paper will be focused on MSC and EPC, since these subsets of cells are the most studied in the field of the cell-based therapies for DM and for diabetic complications.

MSC are a subset of cells that express on their surface CD54/CD102 (intracellular adhesion molecule), CD166 
(vascular cell adhesion molecule), CD49 ( $\alpha$-integrin) as well as CD73 ( $5^{\prime}$ ribonucleotide phosphohydrolase) and CD90 which also regulate cell-to-cell interactions. They also express CD44 (receptor for hyaluronic acid), CD105 (modulator of cellular responses to TGF- $\beta$ ), and MHC1, whereas they do not express CD34, CD14, CD45, CD11a/LFA-1, and CD31, which are surface markers featuring hematopoietic cells and/or EPC instead [4]. MSC are present in the bone marrow, but can also be found in many other fetal and adult tissues. Indeed, they are generally isolated from bone marrow, adipose tissue, umbilical cord blood, and compact bone. MSC display a great therapeutic potential because, beyond their capability to differentiate into muscle, neural precursors, cardiomyocytes, and other cells types, they are able to migrate and home in injured sites, where they act both by regenerating tissues and by secreting trophic factors and paracrine mediators. Moreover, these cells interact with the immune system, particularly with dendritic cells, $\mathrm{T}$ cells and NK cells and therefore they modulate the outcome of immune cells responses, apparently by inhibiting TNF- $\alpha$ and INF- $\gamma$ and by increasing IL-10 [5]. Therefore, their unique immunomodulatory properties make these cells appropriate for both autologous and allogenic transplants, since they avoid and/or actively suppress the immunological responses that cause rejection of transplants. For the same reason, they are now being studied for the treatment of immunological diseases, among which is type $1 \mathrm{DM}$ [6]. Indeed, in the non obese diabetic mice "NOD mice", the injection of MSC reduced the capacity of diabetogenic $\mathrm{T}$ cells to infiltrate pancreatic islets, thus preventing $\beta$-cell destruction [7]. Another model of type $1 \mathrm{DM}$ is injecting mice with streptozotocin, which is a drug destroying the $\beta$-cells [8]. Also in this model, MSC were able to differentiate into insulin-producing cells releasing insulin in a glucose-dependent manner and improving the natural history of diabetes $[9,10]$. Moreover, it has been demonstrated that, when cotransplanted with islets, MSC improved graft morphology and function by the promotion of revascularization [11].

EPC are adult hemangioblast-derived cells [12], which are characterized by the expression of CD34, vascular endothelial growth factor receptor 2 (VEGFR-2), and CD133, which has been included as marker expressed on primitive cells but not on differentiated ones. In fact, as the hemangioblasts destined to become endothelial cells differentiate, they downregulate the hematopoietic cells marker $\mathrm{CD} 133^{+}$ (AC133) expression [12]. EPC can be isolated from human peripheral or umbilical cord blood and can also be found in bone marrow niches. The interest in EPC comes from the fact that these cells have been shown to have direct angiogenic actions and/or to be able to support angiogenesis. Particularly, like for MSC, part of their therapeutic potential could be related to their ability to secrete paracrine mediators. In this respect, several studies have shown that these cells release interleukins, growth factors, and chemokines that altogether regulate $\mathrm{CD} 14^{+}$cells, accelerate vascular network formation, and enhance healing processes [2]. Therefore, they are a promising therapeutic tool in the setting of diabetic complications, which are a consequence of dysfunctional vascular responses.

\section{Rationale for the Use of Adult Stem/Progenitor Cells for Diabetic Complications}

Diabetic patients exhibit impaired mobilization of adult stem cells from the bone marrow [13] and dysfunctional circulating progenitor cells $[14,15]$. A growing body of evidence has demonstrated that DM is associated with a generalized reduction in circulating EPC and that this decline is linearly correlated with the severity of DM, in terms of $\mathrm{HbA1c}$ and blood glucose, whereas it is inversely related to glucose control [16-18]. Busik and colleagues suggested that diabetic neuropathy, altering the circadian rhythm of bone marrow cells release, could be one of the factors accounting for the defective mobilization of stem/progenitor cells coupled to an increased number of cells trapped in the bone marrow [19]. Apart from diabetic neuropathy, the factors that have been classically related to impaired stem/progenitor cells mobilization are the direct and/or indirect effects of hyperglycemia. Fadini and colleagues have demonstrated that the bone marrow mobilization of cells is sensitive to hyperglycemia [13]. Using a model of hind limbs ischemia-reperfusion (I/R) injury for the study of EPC mobilisation in type $1 \mathrm{DM}$, they observed that diabetic rats were completely unable to mobilise EPC after I/R injury, compared to the control rats showing a mobilisation curve within 7 days after injury. However, after insulin administration and premedication with granulocyte-colony stimulating factor (G-CSF) and other stem cells factors, they achieved a partial recovery in postischemic EPC mobilisation [13]. This study suggests that mobilization mechanism is sensitive to chronic hyperglycemia and early on remains reversible.

One of the mechanisms involved in the toxic effects of hyperglycemia on BM-SC seems to be the unbalance between nitric oxide (NO) and reactive oxygen species (ROS) [20]. It is known that hyperglycemia increases ROS formation which, by reacting with $\mathrm{NO}$, lead to a reduction in NO bioavailability, therefore impairing NO signalling. Moreover, diabetic BM-SC display uncoupled endothelial NO synthase (eNOS) activity, promoting the production of ROS and so increasing the unbalance between ROS and NO [20]. Any reduction in NO bioavailability is believed crucial for BMSC mobilization since NO-mediated signalling is essential for activation of MMP-9 which, in turn, shifts resident cells from a quiescent to a proliferative state and stimulates their rapid mobilization into the circulation [21]. Consistent with this concept, Segal and colleagues showed that incubating diabetic CD $34^{+}$cells with NO donors corrected their migratory defect, proving that impaired NO signalling in DM significantly contributes to bone marrow dysfunctional responses [22]. It is reasonable to suggest that MSC migratory properties could also be affected in DM. Diabetic patients display increased circulating levels of osteoprotegerin (OPG) [23], which is a soluble TNF-receptor with atherogenic [24] and diabetogenic [25] actions. Notably this peptide is the decoy receptor for the TNF-related apoptosis-inducing ligand (TRAIL) and displays antiatherosclerotic and antidiabetogenic properties. Our group has recently shown that TRAIL 
is able to promote the migration of BM-MSC in vitro [26]. OPG dose dependently neutralizes the promigratory activity of TRAIL [27], so the high levels of OPG observed in diabetic patients might impair the pro-migratory signalling driven by TRAIL, accounting for the abnormalities of BM-SC in DM.

Several in vitro works have pointed out that the diabetic milieu does not only impair BM-SC mobilization, but it also affects the lifespan and the functions of adult stem cells which may account for the reduction in circulating EPC. Particularly, hyperglycemia has been shown on its own to accelerate the senescence of EPC by the activation of p38/MAPK [28] and Akt/p53/p21 [29] pathways or by downregulation of sirtuin 1 [30]. In this setting, the senescence of EPC could also be due either to the NO reduced bioavailability mentioned previously, since it has been demonstrated that NO delays endothelial cells senescence through the activation of telomerase [31], or to the increased apoptosis induced by ROS. It has indeed been demonstrated that the deletion of p66ShcA, which is a gene regulating the apoptotic responses to oxidative stress, rescues the EPC defects induced by hyperglycemia [32]. However, in a work aimed at defining cross-sectionally the time course of EPC alterations in type 2 DM and to identify potential mechanisms of progenitor cells reduction, Fadini and colleagues found that the lower the count of $\mathrm{CD} 34^{+}$cells the higher their apoptotic rate but also that there was no difference in the apoptotic rate between patients with and without DM and that the percentage of EPC apoptosis was too low to fully explain a decreased cell count [33]. Thus, in vivo studies have not confirmed yet if diabetic EPC have a shortened lifespan, and other mechanisms, apart from the reduced lifespan, may account for the reduction of these cells in DM. Likewise, when cultured in hyperglycemic conditions, MSC increase the production of intracellular ROS which reduce hypoxia-induced factor $1 \alpha$ $(\mathrm{HIF} 1 \alpha)$ expression and consequently attenuate hypoxiainduced vascular endothelial growth factor (VEGF)-A and platelet-derived growth factor (PDGF)-B transcription [34]. Moreover, it is well known that hyperglycemia leads to nonenzymatic glycosylation of proteins and subsequent formation of advanced glycation end products (AGEs) that interacting with their own receptor, RAGE, then activate several intracellular pathways ultimately leading to tissue damage [1]. In this setting, AGEs directly impair the reparative function of both EPC and MSC, and several works have evaluated AGEs deleterious effects on EPC [35-37] as well as on MSC. After isolation of MSC from rats with type $1 \mathrm{DM}$, Stolzing and colleagues studied their ex vivo ability to proliferate and differentiate into the fibroblastic colonyforming unit. They reported that colony size and number were significantly reduced in diabetic rats, mainly because of the induction of cell apoptosis and senescence by AGEs [38]. Consistent with this, when treated with glyceraldehydes and glycolaldehydes, MSC showed reduced cell proliferation, increased cell apoptosis, and impaired differentiation into adipogenic, chondrogenic, and osteogenic clones. These effects were partially prevented by the antiserum against RAGE $[35,39]$.

Altogether these experimental works demonstrate that DM affects the mobilization and the functions of adult stem cells; therefore they provide the rationale for the use of adult stem cells for diabetic complications.

\section{Adult Stem/Progenitor Cells for the Treatment of Macrovascular Complications and Diabetic Cardiomyopathy}

4.1. Macrovascular Complications. Both type 1 and type 2 $\mathrm{DM}$ increase the incidence and progression of atherosclerosis [40] into large arteries and the development of macrovascular complications. Their major clinical manifestations are coronary artery disease (CAD), peripheral artery disease (PAD), and stroke. In particular, patients with DM have a 24 fold increased risk of fatal myocardial infarction, PAD and stroke, together with poorer long-term outcomes [40, 41]. The evidence supporting the utility of cell-based therapies in this setting, and particularly EPC-based therapies, comes from clinical studies showing an inverse relation between the number of EPC and the occurrence of cardiovascular diseases (CVD). Consistent with the reduction of $\mathrm{CD} 133^{+}$cells observed in patients with CVD, CD $34^{+} /$VEGFR-2 ${ }^{+}$and $\mathrm{CD}_{133^{+}}$cells counts have indeed been shown to predict the occurrence of CVD in one-year follow-up studies [42, 43], whilst $\mathrm{CD} 34^{+}$and $\mathrm{CD} 34^{+} / \mathrm{KDR}^{+}$cells counts might be helpful in stratifying the cardiovascular risk of the patients [44]. As expected, in patients with DM and metabolic syndrome, circulating $\mathrm{CD} 34^{+}$cell numbers were also found to be an independent risk marker of CVD [45], leading to the hypothesis that the reduction in circulating progenitors is not only a marker but also a causative factor for the increase in cardiovascular events. Interestingly, significantly lower numbers of EPC were observed in diabetic patients when PAD had developed [46].

Although a study by $\mathrm{Ma}$ and colleagues showed that the treatment with EPC reduced the stenosis obtained after denudation of the common carotid artery in rabbits [47], data on the utility of cell-based therapies to prevent atherosclerosis are indeed conflicting. Silvestre and colleagues have demonstrated that transplantation of BM-SC in ischemic Apolipoprotein E-knockout mice, which is the most largely used animal model for the study of atherosclerosis [41], disappointingly accelerated atherosclerosis without altering the plaque composition [48]. Moreover, smooth muscle progenitor cells have been shown to contribute to the exaggerated intimal hyperplasia found in DM [49]. Consistent with this, in the clinical trials evaluating cellbased therapies after myocardial infarction, one of the major side effects that have been observed was the aggravation of the restenosis [50]. In this setting, another issue that needs to be further investigated is whether arrhythmias are a real safety concern, given that a higher number of arrhythmic events have been reported after intramyocardial delivery of cells, particularly skeletal myoblasts [51]. However, the trials aimed at myocardial repair in patients with acute myocardial infarction have also proven that the intracoronary infusion of $\mathrm{BM}-\mathrm{SC}$ or $\mathrm{CD} 133^{+}$or MSC is associated with an improvement in the global left ventricular ejection fraction, a reduction in the end-systolic left ventricular volumes, and 
a better perfusion in the areas of infarction $[52,53]$. These effects are supposed to be due, at least in part, to the ability of these cells to stimulate myocardial repair/regeneration and neovascularisation (Figure 1).

Cell-based therapies appear promising also in the setting of PAD. A growing body of evidence strongly suggests the utility and effectiveness of adult stem cells for therapeutic neovascularisation both in absence [54-57] and in presence [58-60] of DM. Diabetic PAD is a systemic disease characterized by occlusion of peripheral arteries together with a severe impairment in the development of collateral vessels believed to be caused by endothelial dysfunction and the lack of growth factors, such as VEGF, both driven by glucotoxicity $[1,40]$. The ability of EPC and MSC to produce angiogenic factors (by restoring the physiological levels of VEGF and HIF1 $\alpha$ ) and to differentiate into vascular cells in the periphery [61] has been implicated in the recovery of the native blood flow in ischemic hind limbs after their use. Recently, the transplantation of MSC for therapeutic neovascularisation has also been proven beneficial in type 1 diabetic patients with bilateral upper extremity digital gangrene, demonstrating improved arterial perfusion, good healing of all amputation sites, and cessation of pain [62].

Furthermore, in the context of macrovascular complications, intravenous autologous MSC transplantation has been shown to be able to reduce the mortality rate in patients with ischemic stroke [63].

4.2. Diabetic Cardiomyopathy. Diabetic cardiomyopathy should be considered separately from the so-called macrovascular complications of DM, since it corresponds to the stage characterized by the development of ventricular dysfunction in patients affected by DM, in the absence of CAD, valvular heart disease, or hypertension [64]. Its features, which are heterogeneous, are mainly due to cell apoptosis [64] associated with a dramatic reduction in tissue-resident stem cells [65], extensive myocardial fibrosis, and capillary rarefaction [66]. In particular, it has been shown that the abnormal myocardial matrix deposition associated with DM relies on increased collagen synthesis and on its reduced degradation, whose main effectors are the metalloproteases (MMP). Consistent with this, the diabetic myocardium is characterized by decreased activity of MMP2 , leading to increased collagen accumulation, and increased activity of the apoptotic factor MMP-9 which is responsible for apoptosis of endothelial cells, reduction of capillary density, and poor myocardial perfusion instead. In a study on rats with type $1 \mathrm{DM}$, the intravenous infusion of MSC improved cardiac function through increased angiogenesis and attenuated cardiac remodelling. Eight weeks after the induction of DM, rats were infused with MSC, which then homed into the myocardium and led to increased myocardial arteriolar density and decreased collagen content in the diabetic myocardium. Interestingly, increased MMP-2 activity and decreased transcriptional level of MMP9 were also reported [67]. However, even more fascinating is the possibility of developing noninvasive cell-based therapies relying on the trophic activities of MSC (Figure 1). A recent study with a hamster heart failure model has demonstrated that an intramuscular delivery of MSC would be sufficient to significantly improve ventricular function, enhancing capillary and myocyte densities, attenuating apoptosis, and reducing fibrosis. This was reported to be due to a trophic cross-talk among the injected MSC, the bone marrow, and the heart [68].

\section{Adult Stem/Progenitor Cells for the Treatment of Microvascular Complications and Wound Healing}

5.1. Diabetic Nephropathy. Diabetic nephropathy is now the most common cause of end-stage renal failure in the Western societies. The arterial damages and the changes to the glomerular ultrastructure, mainly mesangial expansion and glomerular membrane thickening, are the principal mechanisms causing diabetic nephropathy [1]. These effects are both driven by hyperglycemia, and thus it is not surprising that one of the most important interventions in preventing diabetic nephropathy, or attenuating it, can be achieved by tight glycemic control [69]. In this setting, it has been shown that EPC mobilize into damaged glomeruli [70], possibly participating in glomerular capillary regeneration. More recently, a subset of hematopoietic stem cells, featured by the expression of the surface molecules CD $24^{+} / \mathrm{CD} 133^{+}$, has been shown promising as it was able to regenerate both tubular cells and podocytes. This is quite significant because the depletion of these cells plays a crucial role in the development of glomerulopathies which are now believed to be podocytopathies [71]. However, in the context of cell-therapy approaches for diabetic nephropathy, the most attractive candidates seem to be the MSC. So far, several works have shown that MSC administration can both prevent and treat diabetic nephropathy. In mice with type 1 DM [72], MSC had the ability to induce $\beta$ pancreatic islets regeneration with consequent achievement of a better glycemic control that, in turn, prevented the development of diabetic nephropathy. MSC also had the ability to slow the progression of diabetic nephropathy through mechanisms independent from glycemic control [73] (Figure 2). Indeed, after an infusion of MSC, $11 \%$ of these cells engrafted into the kidneys, where they differentiated into endothelial cells and possibly mesangial cells. This was associated with a significant decrease in mesangial thickening, extracellular matrix deposition, and macrophages infiltration [74].

5.2. Diabetic Neuropathy. Diabetic neuropathy is estimated to affect over half of the patients with DM. It is a form of neuropathy that affects the somatic and autonomic divisions of the peripheral nervous system, but the spinal cord and the higher central nervous system can also be damaged. The main underlying cause is glucotoxicity and its downstream effects [1]. High glucose levels, oxidative stress, and AGEs reduce nerve blood flow and impair neurotrophic support, altogether leading to neural cells degeneration. Cell-based approaches promoting endogenous production of neurotrophic factors, such as nerve growth factor (NGF), 


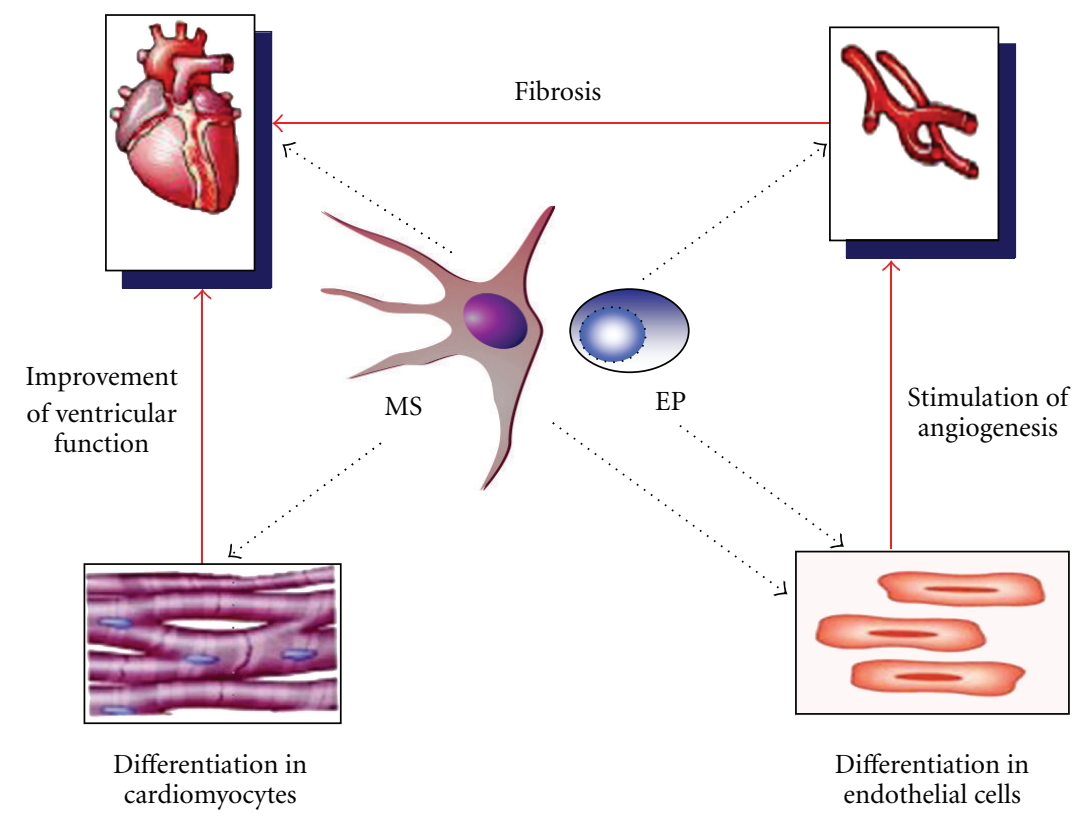

FIGURE 1: Effects of MSC and EPC on myocardial repair/regeneration and angiogenesis. The activities of MSC and EPC may derive from their differentiative ability (into cardiomyocytes and/or endothelial cells) as well as from secretion of paracrine mediators promoting myogenesis, angiogenesis, and heart functionality, in direct and/or indirect manners.

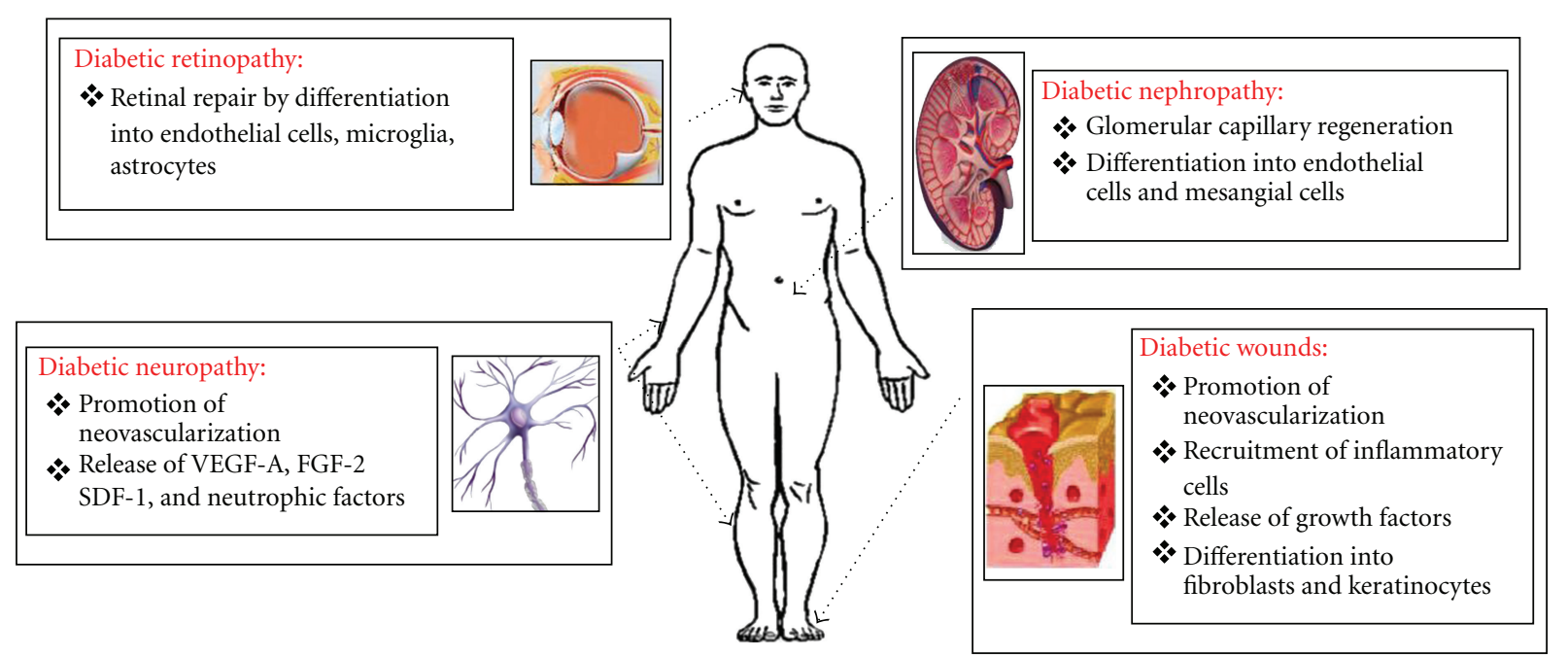

Figure 2: Potential role of EPC and MSC in the control of diabetic microvascular complications and wound healing. Diabetes mellitus is characterized by microvascular complications (retinopathy, nephropathy, and neuropathy) and prolonged/incomplete wound healing. Cell-based therapies may control these complications by different potential mechanisms.

hepatocyte growth factor (HGF), neurotrophin 3, or VEGF, have recently shown some success [1] (Figure 2).

In the setting of diabetic neuropathy, both EPC and MSC have been investigated. Many studies have shown that transplantation of EPC ameliorates the blood flow to peripheral ischemic tissues. Naruse and colleagues investigated whether a unilateral intramuscular injection of EPC into the hind limb skeletal muscles could ameliorate diabetic neuropathy. After such transplantation, more vessels were observed in the injected sites, and this was associated with an amelioration of sciatic nerve blood flow and motor nerve conduction velocity. These data suggest that the ability of EPC to treat diabetic neuropathy is due to the promotion of therapeutic neovascularisation [75] (Figure 2). In addition, in another study, intramuscularly injected EPC not only increased nerve blood flow but also increased the number of vasa nervorum ameliorating the microvascular insufficiency typical of diabetic neuropathy [76]. As a matter of interest, in the same study, EPC were also found to increase the production of VEGF-A, brain-derived neurotrophic factor, fibroblast growth factor (bFGF)-2, stroma-derived factor-1 (SDF-1), and other neurotrophic factors, therefore indicating 
that EPC may reverse various manifestations of diabetic neuropathy through both angiogenic and neurotrophic properties (Figure 2). Consistent with this, in vitro studies proved that EPC were able to make Schwann and endothelial cells proliferate and to reduce the number of apoptotic cells [76].

Also MSC have displayed angiogenic and neurotrophic properties. Four weeks after their intramuscular injection, MSC started producing bFGF and VEGF, and this was associated with an increased ratio between capillaries and muscular fibers, an increased blood flow to the sciatic nerve, an improvement in motor nerve conduction velocity, and a reduced hyperalgesia [77]. Although one of the most exciting properties of MSC, from a therapeutic perspective, is their ability to differentiate into multiple cellular phenotypes, any differentiation into neural cells, such as astrocytes, oligodendrocytes, and Schwann cells, has not yet been observed [77] (Figure 2).

5.3. Diabetic Retinopathy. DM is the leading cause of cases of blindness among adults. Diabetic retinopathy is characterized by a complex of vascular and retinal lesions, all ultimately due to hyperglycemia. This disorder can be categorized into the non proliferative diabetic retinopathy (NPDR) and the proliferative diabetic retinopathy (PDR) [1]. NPRD is characterized by vascular changes leading to retinal ischemia, whereas PDR is the result of an aberrant reactive neovascularisation. Most works carried out in this field have featured EPC, reporting a reduced number of EPC in patients with NPDR [78, 79] but an increased number of EPC in those with PDR. This is consistent with the hypothesis that, since EPC can rescue and maintain the existing retinal capillary bed in healthy patients, the reduced number of EPC observed in DM might predispose to NPDR [2]. Once the damage is initiated, an inflammatory reaction will take place and the bone marrow will respond by increasing the mobilization of EPC, which will eventually result in an abnormal neovascularisation leading to PDR. To date, the studies carried out on ischemic retinal injury have documented the participation of adult stem cells in the retinal repair, showing their ability to home into damaged areas and to differentiate into endothelial cells, microglia, and astrocytes [80-82] (Figure 2). However, these studies were all conducted in animal models of ischemic retinal injury and so concern still remains about the viability of these therapeutic options in the long run, since they could worsen the aberrant reactive neovascularisation featuring the PDR that follows any ischemical retinal injuries. Therefore, PDR may represent a contraindication for angiogenic cell-based therapies.

5.4. Wound Healing. Another common complication of DM is represented by the prolonged and incomplete wound healing, caused by compromised angiogenesis, diminished cells recruitment, lack of growth factors, and impaired formation of collagen matrix. It has been demonstrated that generally the number of MSC increases considerably in the site of an injury, and that after a vascular trauma a rapid mobilization to the injured site of EPC also takes place.
Wound healing normally results from a combined effort of inflammatory and noninflammatory cells recruited to the injured site. Recent studies suggest that MSC and EPC are a significant proportion of the noninflammatory cells that migrate to the skin. In DM, the number of EPC within the granulation tissue has been found to be significantly reduced with respect to non diabetic controls [83] and locally increased apoptosis and decreased proliferation of these cells have also been reported. Several works have shown that MSC accelerate wound closure by differentiating into fibroblasts and keratinocytes, and promoting neovascularisation and regeneration of appendages and recruiting inflammatory cells into wounds $[84,85]$ (Figure 2). Transplantation of EPC has also been shown to enhance wound healing in mice [86], and this seems to rely on the release of paracrine mediators, such as the release of VEGF, HGF, G-CSF, and PDGF [84, 87]. As expected, in the setting of DM, the same mechanisms, mentioned above for MSC and EPC, have been shown to enhance wound healing $[88,89]$ and to also be an effective treatment of foot ulcerations [90-92] (Figure 2).

\section{Genetic Manipulation and Pharmacological Strategies Aimed at Reversing the Alterations of Adult Stem/Progenitor Cells in Diabetes}

6.1. Genetic Manipulation. The evidence obtained so far makes for a compelling argument for the use of MSC and/or EPC in the setting of DM [2]. Because of the broadly dysfunctional cell functions found in DM, it is believed that cells to be used for treatment of diabetic complications should be equipped with cellular and molecular tools to make them withstand the in vivo diabetic milieu. Thus, studies into the genetic modification and/or manipulation of diabetic cells have commenced as approaches in overcoming this issue. In recent work, Marrotte and colleagues [93] transfected EPC with the gene of manganese superoxide dismutase, in order to correct its decreased expression found in diabetic EPC. They found that, after this ex vivo manipulation, the EPC transplanted contributed significantly to the accelerated wound healing in a type 2 DM animal model. So far, several molecules have been targeted, such as human telomerase reverse transcriptase (hTERT), which was shown to delay EPC senescence [94], and the glycogen synthase kinase 3$\beta$, which enhanced the EPC vasoregenerative potential [95]. In MSC, the overexpression of GATA-4, CXCR4, and Akt-1 led, respectively, to increased cell survival and angiogenesis [96], enhanced in vivo mobilization into ischemic areas [97], and better functional repair in a mouse infarct model [98]. Although the genetic manipulation of adult stem cells dysfunctions in DM has shown promising results, one should be very cautious when adopting this approach because of its potential side effects. For instance, targeting senescence/survival regulatory pathways warrants greater understanding given the risk of malignant transformation of the cells.

6.2. Pharmacological Strategies. Other approaches have pharmacologically targeted the intracellular dysfunctions that 
take place in DM. For example, the effects of AVE9488 [99], GH [100], both stimulating eNOS, and those of rosiglitazone, which has antioxidant properties [101] have been studied as treatments for the reduced NO bioavailability [102]. Interestingly, Sorrentino and colleagues showed that the effect of rosiglitazone treatment was comparable to that of small-interfering RNA silencing NADPH oxidase subunit p47. Both approaches reduced NADPH oxidase activity, restoring NO bioavailability, and improved in vivo reendothelization capacity of EPC isolated from diabetic patients. However, whether increasing $\mathrm{NO}$ production and bioavailability may result in higher production of reactive oxygen species that will further increase oxidative stress leading to vascular damage is unknown yet. The blockade of p38/MAPK pathway, using its specific inhibitor SB203580, has also been assayed. Seeger and colleagues demonstrated that the ex vivo treatment of EPC with SB203580 was able to significantly ameliorate their revascularisation properties, possibly through the regulation of their proliferation and differentiation [103]. Ex vivo treatment of MSC with IGF1 and IGF-2 made MSC regain the functions affected by DM [104]. Finally, antagonists of CXCR4 (such as AMD3100 and SDF-1 $\beta$ P2G), which disrupt the interaction between the CXCR4 receptor (on hematopoietic cells) and the CXCL12 (expressed by stromal cells), have already been shown promising in accelerating blood flow restoration in diabetic mice [105].

\section{Conclusions}

The past decade has provided new and fascinating in vitro and in vivo data supporting the use of MSC and EPC for the treatment of diabetic complications. However, among the issues raised, the possible contribution of these cells to lesion formation, in terms of atherogenesis, neointimal hyperplasia, and retinal aberrant angiogenesis, as well as the potential risk of their malignant transformation will certainly require further long-term analysis. Also, it is yet to define the best way to make these cells withstand the diabetic milieu in the long run. Therefore, a greater understanding of MSC and EPC biology, both in in vitro and in vivo studies, is needed to establish the safety of their use as a novel and efficient therapeutic agents in the treatment of complications of DM.

\section{References}

[1] N. A. Calcutt, M. E. Cooper, T. S. Kern, and A. M. Schmidt, "Therapies for hyperglycaemia-induced diabetic complications: from animal models to clinical trials," Nature Reviews Drug Discovery, vol. 8, no. 5, pp. 417-429, 2009.

[2] Y. P. Jarajapu and M. B. Grant, "The promise of cellbased therapies for diabetic complications: challenges and solutions," Circulation Research, vol. 106, no. 5, pp. 854-869, 2010.

[3] T. Asahara, T. Murohara, A. Sullivan et al., "Isolation of putative progenitor endothelial cells for angiogenesis," Science, vol. 275, no. 5302, pp. 964-967, 1997.

[4] V. Volarevic, N. Arsenijevic, M. L. Lukic, and M. Stojkovic, "Mesenchymal stem cell treatment of complications of diabetes mellitus," Stem Cells, vol. 29, no. 1, pp. 5-10, 2011.
[5] S. Aggarwal and M. F. Pittenger, "Human mesenchymal stem cells modulate allogeneic immune cell responses," Blood, vol. 105, no. 4, pp. 1815-1822, 2005.

[6] P. Fiorina, M. Jurewicz, A. Augello et al., "Immunomodulatory function of bone marrow-derived mesenchymal stem cells in experimental autoimmune type 1 diabetes," Journal of Immunology, vol. 183, no. 2, pp. 993-1004, 2009.

[7] A. M. Madec, R. Mallone, G. Afonso et al., "Mesenchymal stem cells protect NOD mice from diabetes by inducing regulatory T cells," Diabetologia, vol. 52, no. 7, pp. 1391$1399,2009$.

[8] G. Zauli, B. Toffoli, M. G. Di Iasio, C. Celeghini, B. Fabris, and P. Secchiero, "Treatment with recombinant tumor necrosis factor-related apoptosis-inducing ligand alleviates the severity of streptozotocin-induced diabetes," Diabetes, vol. 59, no. 5, pp. 1261-1265, 2010.

[9] Q. P. Xie, H. Huang, B. Xu et al., "Human bone marrow mesenchymal stem cells differentiate into insulin-producing cells upon microenvironmental manipulation in vitro," Differentiation, vol. 77, no. 5, pp. 483-491, 2009.

[10] Q. Y. Dong, L. Chen, G. Q. Gao et al., "Allogeneic diabetic mesenchymal stem cells transplantation in streptozotocininduced diabetic rat," Clinical and Investigative Medicine, vol. 31, no. 6, pp. E328-E337, 2008.

[11] T. Ito, S. Itakura, I. Todorov et al., "Mesenchymal stem cell and islet co-transplantation promotes graft revascularization and function," Transplantation, vol. 89, no. 12, pp. 14381445, 2010.

[12] G. C. Schatteman, O. Awad et al., "Hemangioblasts, angioblasts, and adult endothelial cell progenitors," Anatomical Record-Part A Discoveries in Molecular, Cellular, and Evolutionary Biology, vol. 276, no. 1, pp. 13-21, 2004.

[13] G. P. Fadini, S. Sartore, M. Schiavon et al., "Diabetes impairs progenitor cell mobilisation after hindlimb ischaemiareperfusion injury in rats," Diabetologia, vol. 49, no. 12, pp. 3075-3084, 2006.

[14] C. J. M. Loomans, E. J. P. De Koning, F. J. T. Staal et al., "Endothelial progenitor cell dysfunction: a novel concept in the pathogenesis of vascular complications of type 1 diabetes," Diabetes, vol. 53, no. 1, pp. 195-199, 2004.

[15] O. M. Tepper, R. D. Galiano, J. M. Capla et al., "Human endothelial progenitor cells from type II diabetics exhibit impaired proliferation, adhesion, and incorporation into vascular structures," Circulation, vol. 106, no. 22, pp. 27812786, 2002.

[16] T. Kusuyama, T. Omura, D. Nishiya et al., "Effects of treatment for diabetes mellitus on circulating vascular progenitor cells," Journal of Pharmacological Sciences, vol. 102, no. 1, pp. 96-102, 2006.

[17] G. P. Fadini, L. Pucci, R. Vanacore et al., "Glucose tolerance is negatively associated with circulating progenitor cell levels," Diabetologia, vol. 50, no. 10, pp. 2156-2163, 2007.

[18] C. G. Egan, R. Lavery, F. Caporali et al., "Generalised reduction of putative endothelial progenitors and CXCR4-positive peripheral blood cells in type 2 diabetes," Diabetologia, vol. 51, no. 7, pp. 1296-1305, 2008.

[19] J. V. Busik, M. Tikhonenko, A. Bhatwadekar et al., "Diabetic retinopathy is associated with bone marrow neuropathy and a depressed peripheral clock," Journal of Experimental Medicine, vol. 206, no. 13, pp. 2897-2906, 2009.

[20] T. Thum, D. Fraccarollo, M. Schultheiss et al., "Endothelial nitric oxide synthase uncoupling impairs endothelial progenitor cell mobilization and function in diabetes," Diabetes, vol. 56, no. 3, pp. 666-674, 2007. 
[21] B. Heissig, K. Hattori, S. Dias et al., "Recruitment of stem and progenitor cells from the bone marrow niche requires MMP-9 mediated release of Kit-ligand," Cell, vol. 109, no. 5, pp. 625-637, 2002.

[22] M. S. Segal, R. Shah, A. Afzal et al., "Nitric oxide cytoskeletalinduced alterations reverse the endothelial progenitor cell migratory defect associated with diabetes," Diabetes, vol. 55, no. 1, pp. 102-109, 2006.

[23] W. S. Browner, L. Y. Lui, and S. R. Cummings, "Associations of serum osteoprotegerin levels with diabetes, stroke, bone density, fractures, and mortality in elderly women," Journal of Clinical Endocrinology and Metabolism, vol. 86, no. 2, pp. 631-637, 2001.

[24] R. Candido, B. Toffoli, F. Corallini et al., "Human full-length osteoprotegerin induces the proliferation of rodent vascular smooth muscle cells both in vitro and in vivo," Journal of Vascular Research, vol. 47, no. 3, pp. 252-261, 2010.

[25] B. Toffoli, S. Bernardi, R. Candido et al., "Osteoprotegerin induces morphological and functional alterations in mouse pancreatic islets," Molecular and Cellular Endocrinology, vol. 331, no. 1, pp. 136-142, 2011.

[26] P. Secchiero, E. Melloni, F. Corallini et al., "Tumor necrosis factor-related apoptosis-inducing ligand promotes migration of human bone marrow multipotent stromal cells," Stem Cells, vol. 26, no. 11, pp. 2955-2963, 2008.

[27] F. Corallini, P. Secchiero, A. P. Beltrami et al., "TNF- $\alpha$ modulates the migratory response of mesenchymal stem cells to TRAIL," Cellular and Molecular Life Sciences, vol. 67, no. 8, pp. 1307-1314, 2010.

[28] S. Kuki, T. Imanishi, K. Kobayashi, Y. Matsuo, M. Obana, and T. Akasaka, "Hyperglycemia accelerated endothelial progenitor cell senescence via the activation of p38 mitogenactivated protein kinase," Circulation Journal, vol. 70, no. 8, pp. 1076-1081, 2006.

[29] A. Rosso, A. Balsamo, R. Gambino et al., "p53 mediates the accelerated onset of senescence of endothelial progenitor cells in diabetes," Journal of Biological Chemistry, vol. 281, no. 7, pp. 4339-4347, 2006.

[30] M. L. Balestrieri, M. Rienzo, F. Felice et al., "High glucose downregulates endothelial progenitor cell number via SIRT1," Biochimica et Biophysica Acta, vol. 1784, no. 6, pp. 936-945, 2008.

[31] M. Vasa, K. Breitschopf, A. M. Zeiher, and S. Dimmeler, "Nitric oxide activates telomerase and delays endothelial cell senescence," Circulation Research, vol. 87, no. 7, pp. 540-542, 2000.

[32] V. Di Stefano, C. Cencioni, G. Zaccagnini, A. Magenta, M. C. Capogrossi, and F. Martelli, "P66 ShcA modulates oxidative stress and survival of endothelial progenitor cells in response to high glucose," Cardiovascular Research, vol. 82, no. 3, pp. 421-429, 2009.

[33] G. P. Fadini, E. Boscaro, S. De Kreutzenberg et al., "Time course and mechanisms of circulating progenitor cell reduction in the natural history of type 2 diabetes," Diabetes Care, vol. 33, no. 5, pp. 1097-1102, 2010.

[34] T. Ishizuka, T. Hinata, and Y. Watanabe, "Superoxide induced by a high-glucose concentration attenuates production of angiogenic growth factors in hypoxic mouse mesenchymal stem cells," Journal of Endocrinology, vol. 208, no. 2, pp. 147159, 2011.

[35] Q. Chen, L. Dong, L. Wang, L. Kang, and B. Xu, "Advanced glycation end products impair function of late endothelial progenitor cells through effects on protein kinase Akt and cyclooxygenase-2," Biochemical and Biophysical Research Communications, vol. 381, no. 2, pp. 192-197, 2009.

[36] C. Sun, C. Liang, Y. Ren et al., "Advanced glycation end products depress function of endothelial progenitor cells via p38 and ERK 1/2 mitogen-activated protein kinase pathways," Basic Research in Cardiology, vol. 104, no. 1, pp. 42-49, 2009.

[37] R. J. Scheubel, S. Kahrstedt, H. Weber et al., "Depression of progenitor cell function by advanced glycation endproducts (AGEs): potential relevance for impaired angiogenesis in advanced age and diabetes," Experimental Gerontology, vol. 41, no. 5, pp. 540-548, 2006.

[38] A. Stolzing, D. Sellers, O. Llewelyn, and A. Scutt, "Diabetes induced changes in rat mesenchymal stem cells," Cells Tissues Organs, vol. 191, no. 6, pp. 453-465, 2010.

[39] S. Kume, S. Kato, S. I. Yamagishi et al., "Advanced glycation end-products attenuate human mesenchymal stem cells and prevent cognate differentiation into adipose tissue, cartilage, and bone," Journal of Bone and Mineral Research, vol. 20, no. 9, pp. 1647-1658, 2005.

[40] J. A. Beckman, M. A. Creager, and P. Libby, "Diabetes and atherosclerosis epidemiology, pathophysiology, and management," Journal of the American Medical Association, vol. 287, no. 19 , pp. 2570-2581, 2002.

[41] P. Secchiero, R. Candido, F. Corallini et al., "Systemic tumor necrosis factor-related apoptosis-inducing ligand delivery shows antiatherosclerotic activity in apolipoprotein E-null diabetic mice," Circulation, vol. 114, no. 14, pp. 1522-1530, 2006.

[42] C. Schmidt-Lucke, L. Rössig, S. Fichtlscherer et al., "Reduced number of circulating endothelial progenitor cells predicts future cardiovascular events: proof of concept for the clinical importance of endogenous vascular repair," Circulation, vol. 111, no. 22, pp. 2981-2987, 2005.

[43] N. Werner, S. Kosiol, T. Schiegl et al., "Circulating endothelial progenitor cells and cardiovascular outcomes," The New England Journal of Medicine, vol. 353, no. 10, pp. 999-1007, 2005.

[44] G. P. Fadini, S. Maruyama, T. Ozaki et al., "Circulating progenitor cell count for cardiovascular risk stratification: a pooled analysis," PLoS ONE, vol. 5, no. 7, Article ID e11488, 2010.

[45] G. P. Fadini, S. Sartore, M. Albiero et al., "Number and function of endothelial progenitor cells as a marker of severity for diabetic vasculopathy," Arteriosclerosis, Thrombosis, and Vascular Biology, vol. 26, no. 9, pp. 2140-2146, 2006.

[46] G. P. Fadini, M. Miorin, M. Facco et al., "Circulating endothelial progenitor cells are reduced in peripheral vascular complications of type 2 diabetes mellitus," Journal of the American College of Cardiology, vol. 45, no. 9, pp. 1449-1457, 2005.

[47] Z. L. Ma, X. L. Mai, J. H. Sun et al., "Inhibited atherosclerotic plaque formation by local administration of magnetically labeled endothelial progenitor cells (EPCs) in a rabbit model," Atherosclerosis, vol. 205, no. 1, pp. 80-86, 2009.

[48] J. S. Silvestre, A. Gojova, V. Brun et al., "Transplantation of bone marrow-derived mononuclear cells in ischemic apolipoprotein e-Knockout mice accelerates atherosclerosis without altering plaque composition," Circulation, vol. 108, no. 23, pp. 2839-2842, 2003.

[49] P. E. Westerweel, C. T. J. van Velthoven, T. Q. Nguyen et al., "Modulation of TGF- $\beta /$ BMP-6 expression and increased levels of circulating smooth muscle progenitor cells in a type 
I diabetes mouse model," Cardiovascular Diabetology, vol. 9, p. 55, 2010.

[50] H. J. Kang, H. S. Kim, S. Y. Zhang et al., "Effects of intracoronary infusion of peripheral blood stem-cells mobilised with granulocyte-colony stimulating factor on left ventricular systolic function and restenosis after coronary stenting in myocardial infarction: the MAGIC cell randomised clinical trial," The Lancet, vol. 363, no. 9411, pp. 751-756, 2004.

[51] P. Menasché, "Stem cell therapy for heart failure: are arrhythmias a real safety concern?" Circulation, vol. 119, no. 20, pp. 2735-2740, 2009.

[52] S. Dimmeler, J. Burchfield, and A. M. Zeiher, "Cell-based therapy of myocardial infarction," Arteriosclerosis, Thrombosis, and Vascular Biology, vol. 28, no. 2, pp. 208-216, 2008.

[53] J. M. Hare, J. H. Traverse, T. D. Henry et al., "A randomized, double-blind, placebo-controlled, dose-escalation study of intravenous adult human mesenchymal stem cells (prochymal) after acute myocardial infarction," Journal of the American College of Cardiology, vol. 54, no. 24, pp. 22772286, 2009.

[54] T. Takahashi, C. Kalka, H. Masuda et al., "Ischemiaand cytokine-induced mobilization of bone marrow-derived endothelial progenitor cells for neovascularization," Nature Medicine, vol. 5, no. 4, pp. 434-438, 1999.

[55] T. Murohara, H. Ikeda, J. Duan et al., "Transplanted cord blood-derived endothelial precursor cells augment postnatal neovascularization," Journal of Clinical Investigation, vol. 105, no. 11, pp. 1527-1536, 2000.

[56] C. Kalka, H. Masuda, T. Takahashi et al., "Transplantation of ex vivo expanded endothelial progenitor cells for therapeutic neovascularization," Proceedings of the National Academy of Sciences of the United States of America, vol. 97, no. 7, pp. 3422-3427, 2000.

[57] G. P. Fadini, C. Agostini, and A. Avogaro, "Autologous stem cell therapy for peripheral arterial disease. Meta-analysis and systematic review of the literature," Atherosclerosis, vol. 209, no. 1, pp. 10-17, 2010.

[58] G. C. Schatteman, H. D. Hanlon, C. Jiao, S. G. Dodds, and B. A. Christy, "Blood-derived angioblasts accelerate blood-flow restoration in diabetic mice," Journal of Clinical Investigation, vol. 106, no. 4, pp. 571-578, 2000.

[59] K. Hirata, T. S. Li, M. Nishida et al., "Autologous bone marrow cell implantation as therapeutic angiogenesis for ischemic hindlimb in diabetic rat model," American Journal of Physiology, vol. 284, no. 1, pp. H66-H70, 2003.

[60] A. H. Amin, Z. Y. Abd Elmageed, D. Nair et al., "Modified multipotent stromal cells with epidermal growth factor restore vasculogenesis and blood flow in ischemic hind-limb of type II diabetic mice," Laboratory Investigation, vol. 90, no. 7, pp. 985-996, 2010.

[61] U. M. Gehling, S. Ergün, U. Schumacher et al., "In vitro differentiation of endothelial cells from AC133-positive progenitor cells," Blood, vol. 95, no. 10, pp. 3106-3112, 2000.

[62] A. J. Comerota, A. Link, J. Douville, and E. R. Burchardt, "Upper extremity ischemia treated with tissue repair cells from adult bone marrow," Journal of Vascular Surgery, vol. 52, no. 3, pp. 723-729, 2010.

[63] J. S. Lee, J. M. Hong, G. J. Moon, P. H. Lee, Y. H. Ahn, and O. Y. Bang, "A long-term follow-up study of intravenous autologous mesenchymal stem cell transplantation in patients with ischemic stroke," Stem Cells, vol. 28, no. 6, pp. 1099-1106, 2010 .
[64] B. Toffoli, S. Bernardi, R. Candido, S. Zacchigna, B. Fabris, and P. Secchiero, "TRAIL shows potentialcardioprotective activity," Investigational New Drugs. In press.

[65] M. Rota, N. LeCapitaine, T. Hosoda et al., "Diabetes promotes cardiac stem cell aging and heart failure, which are prevented by deletion of the p66shc gene," Circulation Research, vol. 99, no. 1, pp. 42-52, 2006.

[66] Y. S. Yoon, S. Uchida, O. Masuo et al., "Progressive attenuation of myocardial vascular endothelial growth factor expression is a seminal event in diabetic cardiomyopathy: restoration of microvascular homeostasis and recovery of cardiac function in diabetic cardiomyopathy after replenishment of local vascular endothelial growth factor," Circulation, vol. 111, no. 16, pp. 2073-2085, 2005.

[67] N. Zhang, J. Li, R. Luo, J. Jiang, and J. A. Wang, "Bone marrow mesenchymal stem cells induce angiogenesis and attenuate the remodeling of diabetic cardiomyopathy," Experimental and Clinical Endocrinology and Diabetes, vol. 116, no. 2, pp. 104-111, 2008.

[68] A. Shabbir, D. Zisa, G. Suzuki, and T. Lee, "Heart failure therapy mediated by the trophic activities of bone marrow mesenchymal stem cells: a noninvasive therapeutic regimen," American Journal of Physiology, vol. 296, no. 6, pp. H1888H1897, 2009.

[69] M. E. Cooper, "Pathogenesis, prevention, and treatment of diabetic nephropathy," Lancet, vol. 352, no. 9123, pp. 213219, 1998.

[70] Y. Abe-Yoshio, K. Abe, M. Miyazaki et al., "Involvement of bone marrow-derived endothelial progenitor cells in glomerular capillary repair in habu snake venom-induced glomerulonephritis," Virchows Archiv, vol. 453, no. 1, pp. 97106, 2008.

[71] E. Ronconi, C. Sagrinati, M. L. Angelotti et al., "Regeneration of glomerular podocytes by human renal progenitors," Journal of the American Society of Nephrology, vol. 20, no. 2, pp. 322-332, 2009.

[72] F. E. Ezquer, M. E. Ezquer, D. B. Parrau, D. Carpio, A. J. Yañez, and P. A. Conget, "Systemic administration of multipotent mesenchymal stromal cells reverts hyperglycemia and prevents nephropathy in type 1 diabetic mice," Biology of Blood and Marrow Transplantation, vol. 14, no. 6, pp. 631640, 2008.

[73] F. Ezquer, M. Ezquer, V. Simon et al., "Endovenous administration of bone marrow-derived multipotent mesenchymal stromal cells prevents renal failure in diabetic mice," Biology of Blood and Marrow Transplantation, vol. 15, no. 11, pp. 1354-1365, 2009.

[74] R. H. Lee, M. J. Seo, R. L. Reger et al., "Multipotent stromal cells from human marrow home to and promote repair of pancreatic islets and renal glomeruli in diabetic NOD/scid mice," Proceedings of the National Academy of Sciences of the United States of America, vol. 103, no. 46, pp. 17438-17443, 2006.

[75] K. Naruse, Y. Hamada, E. Nakashima et al., "Therapeutic neovascularization using cord blood-derived endothelial progenitor cells for diabetic neuropathy," Diabetes, vol. 54, no. 6, pp. 1823-1828, 2005.

[76] J. O. Jeong, M. O. Kim, H. Kim et al., "Dual angiogenic and neurotrophic effects of bone marrow-derived endothelial progenitor cells on diabetic neuropathy," Circulation, vol. 119, no. 5, pp. 699-708, 2009.

[77] T. Shibata, K. Naruse, H. Kamiya et al., "Transplantation of bone marrow-derived mesenchymal stem cells improves 
diabetic polyneuropathy in rats," Diabetes, vol. 57, no. 11, pp. 3099-3107, 2008.

[78] S. Brunner, G. H. Schernthaner, M. Satler et al., "Correlation of different circulating endothelial progenitor cells to stages of diabetic retinopathy: first in vivo data," Investigative Ophthalmology and Visual Science, vol. 50, no. 1, pp. 392-398, 2009.

[79] I. G. Lee, S. L. Chae, and J. C. Kim, "Involvement of circulating endothelial progenitor cells and vasculogenic factors in the pathogenesis of diabetic retinopathy," Eye, vol. 20, no. 5, pp. 546-552, 2006.

[80] M. B. Grant, W. S. May, S. Caballero et al., "Adult hematopoietic stem cells provide functional hemangioblast activity during retinal neovascularization," Nature Medicine, vol. 8, no. 6, pp. 607-612, 2002.

[81] M. R. Ritter, E. Banin, S. K. Moreno, E. Aguilar, M. I. Dorrell, and M. Friedlander, "Myeloid progenitors differentiate into microglia and promote vascular repair in a model of ischemic retinopathy," Journal of Clinical Investigation, vol. 116, no. 12, pp. 3266-3276, 2006.

[82] Z. Yang, K. Li, X. Yan, F. Dong, and C. Zhao, "Amelioration of diabetic retinopathy by engrafted human adipose-derived mesenchymal stem cells in streptozotocin diabetic rats," Graefe's Archive for Clinical and Experimental Ophthalmology, vol. 248, no. 10, pp. 1415-1422, 2010.

[83] M. Albiero, L. Menegazzo, E. Boscaro, C. Agostini, A. Avogaro, and G. P. Fadini, "Defective recruitment, survival and proliferation of bone marrow-derived progenitor cells at sites of delayed diabetic wound healing in mice," Diabetologia, vol. 54, no. 4, pp. 945-953, 2011.

[84] Y. Wu, R. C. H. Zhao, and E. E. Tredget, "Concise review: bone marrow-derived stem/progenitor cells in cutaneous repair and regeneration," Stem Cells, vol. 28, no. 5, pp. 905915, 2010.

[85] M. Gill, S. Dias, K. Hattori et al., "Vascular trauma induces rapid but transient mobilization of VEGFR2 ${ }^{+} \mathrm{AC} 33^{+}$ endothelial precursor cells," Circulation Research, vol. 88, no. 2, pp. 167-174, 2001.

[86] W. Suh, K. L. Kim, J. M. Kim et al., "Transplantation of endothelial progenitor cells accelerates dermal wound healing with increased recruitment of monocytes/macrophages and neovascularization," Stem Cells, vol. 23, no. 10, pp. 15711578, 2005.

[87] J. Rehman, J. Li, C. M. Orschell, and K. L. March, "Peripheral blood "endothelial progenitor cells" are derived from monocyte/macrophages and secrete angiogenic growth factors," Circulation, vol. 107, no. 8, pp. 1164-1169, 2003.

[88] Y. Wu, L. Chen, P. G. Scott, and E. E. Tredget, "Mesenchymal stem cells enhance wound healing through differentiation and angiogenesis," Stem Cells, vol. 25, no. 10, pp. 2648-2659, 2007.

[89] D. S. Kwon, X. Gao, Y. B. Liu et al., "Treatment with bone marrow-derived stromal cells accelerates wound healing in diabetic rats," International Wound Journal, vol. 5, no. 3, pp. 453-463, 2008.

[90] J. Vojtaššák, L. Danišovič, M. Kubeš et al., "Autologous biograft and mesenchymal stem cells in treatment of the diabetic foot," Neuroendocrinology Letters, vol. 27, supplement 2, pp. 134-137, 2006.

[91] E. Sivan-Loukianova, O. A. Awad, V. Stepanovic, J. Bickenbach, and G. C. Schatteman, "CD34+ blood cells accelerate vascularization and healing of diabetic mouse skin wounds," Journal of Vascular Research, vol. 40, no. 4, pp. 368-377, 2003.

[92] L. S. Barcelos, C. Duplaa, N. Kränkel et al., "Human CD133+ progenitor cells promote the healing of diabetic ischemic ulcers by paracrine stimulation of angiogenesis and activation of Wnt signaling," Circulation Research, vol. 104, no. 9, pp. 1095-1102, 2009.

[93] E. J. Marrotte, D. D. Chen, J. S. Hakim, and A. F. Chen, "Manganese superoxide dismutase expression in endothelial progenitor cells accelerates wound healing in diabetic mice," Journal of Clinical Investigation, vol. 120, no. 12, pp. 42074219, 2010

[94] S. Murasawa, J. Llevadot, M. Silver, J. M. Isner, D. W. Losordo, and T. Asahara, "Constitutive human telomerase reverse transcriptase expression enhances regenerative properties of endothelial progenitor cells," Circulation, vol. 106, no. 9, pp. 1133-1139, 2002.

[95] J. H. Choi, J. Hurt, C. H. Yoon et al., "Augmentation of therapeutic augiogenesis using genetically modified human endothelial progenitor cells with altered glycogen synthase kinase- $3 \beta$ activity," Journal of Biological Chemistry, vol. 279, no. 47, pp. 49430-49438, 2004.

[96] H. Li, S. Zuo, Z. He et al., "Paracrine factors released by GATA- 4 overexpressed mesenchymal stem cells increase angiogenesis and cell survival," American Journal of Physiology, vol. 299, no. 6, pp. H1772-H1781, 2010.

[97] D. Zhang, G. C. Fan, X. Zhou et al., "Over-expression of CXCR4 on mesenchymal stem cells augments myoangiogenesis in the infarcted myocardium," Journal of Molecular and Cellular Cardiology, vol. 44, no. 2, pp. 281-292, 2008.

[98] A. A. Mangi, N. Noiseux, D. Kong et al., "Mesenchymal stem cells modified with Akt prevent remodeling and restore performance of infarcted hearts," Nature Medicine, vol. 9, no. 9, pp. 1195-1201, 2003.

[99] K. I. Sasaki, C. Heeschen, A. Aicher et al., "Ex vivo pretreatment of bone marrow mononuclear cells with endothelial NO synthase enhancer AVE9488 enhances their functional activity for cell therapy," Proceedings of the National Academy of Sciences of the United States of America, vol. 103, no. 39, pp. 14537-14541, 2006.

[100] T. Thum, F. Fleissner, I. Klink et al., "Growth hormone treatment improves markers of systemic nitric oxide bioavailability via insulin-like growth factor-I," Journal of Clinical Endocrinology and Metabolism, vol. 92, no. 11, pp. 41724179, 2007.

[101] G. Ceolotto, A. Gallo, I. Papparella et al., "Rosiglitazone reduces glucose-induced oxidative stress mediated by $\mathrm{NAD}(\mathrm{P}) \mathrm{H}$ oxidase via AMPK-dependent mechanism," Arteriosclerosis, Thrombosis, and Vascular Biology, vol. 27, no. 12, pp. 2627-2633, 2007.

[102] S. A. Sorrentino, F. H. Bahlmann, C. Besler et al., "Oxidant stress impairs in vivo reendothelialization capacity of endothelial progenitor cells from patients with type 2 diabetes mellitus: restoration by the peroxisome proliferatoractivated receptor- $\gamma$ agonist rosiglitazone," Circulation, vol. 116, no. 2, pp. 163-173, 2007.

[103] F. H. Seeger, J. Haendeler, D. H. Walter et al., "p38 mitogen-activated protein kinase downregulates endothelial progenitor cells," Circulation, vol. 111, no. 9, pp. 1184-1191, 2005.

[104] M. Khan, S. Akhtar, S. Mohsin, N. Khan, and S. Riazuddin, "Growth factor preconditioning increases the function of diabetes-impaired mesenchymal stem cells," Stem Cells and Development, vol. 20, no. 1, pp. 67-75, 2011.

[105] C. Jiao, S. Fricker, and G. C. Schatteman, "The chemokine (C-X-C motif) receptor 4 inhibitor AMD3100 accelerates blood flow restoration in diabetic mice," Diabetologia, vol. 49, no. 11, pp. 2786-2789, 2006. 


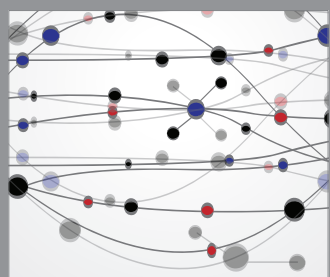

The Scientific World Journal
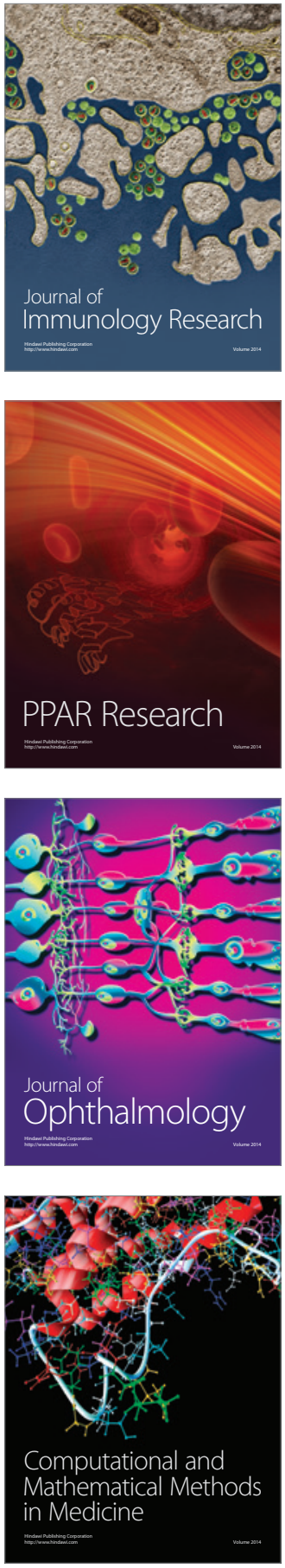

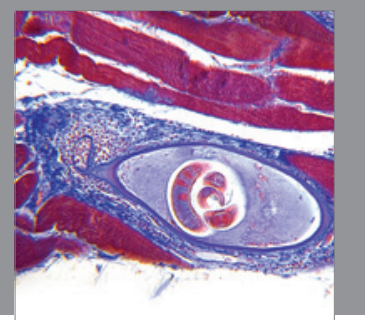

Gastroenterology

Research and Practice
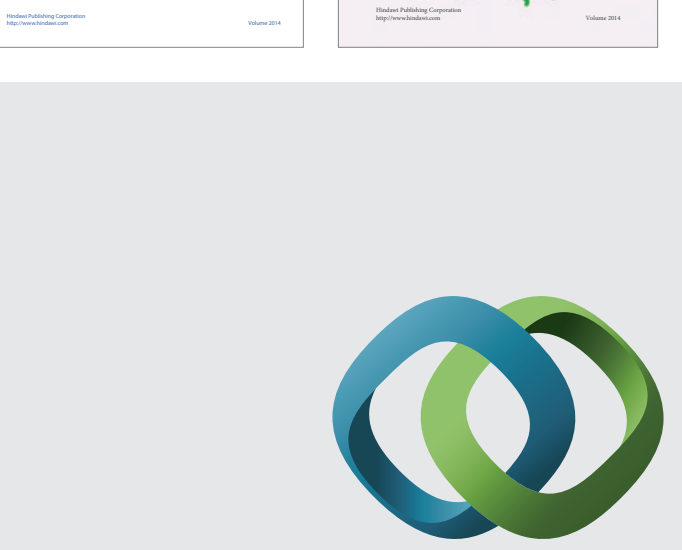

\section{Hindawi}

Submit your manuscripts at

http://www.hindawi.com
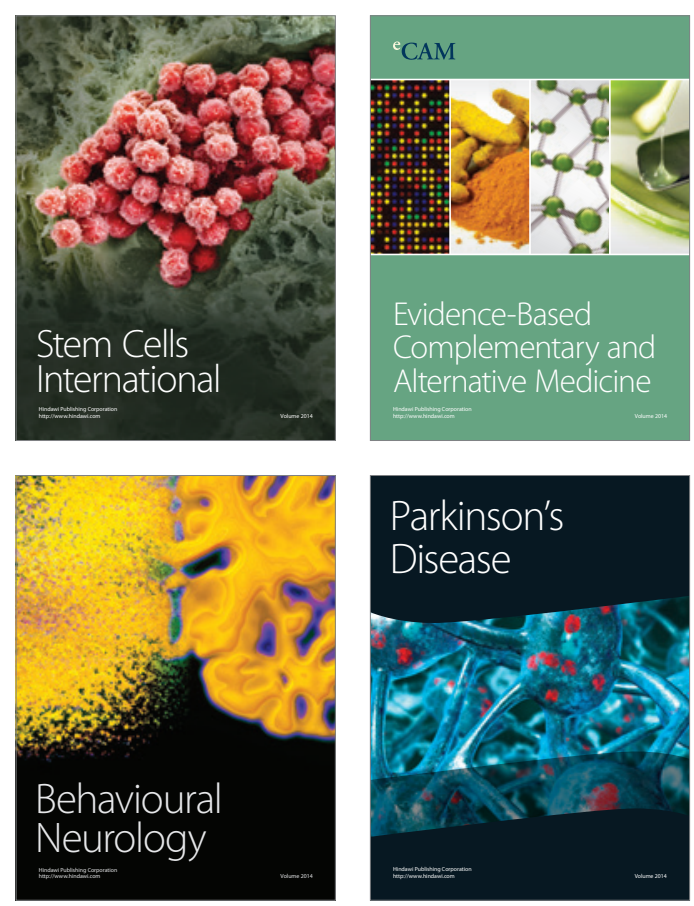

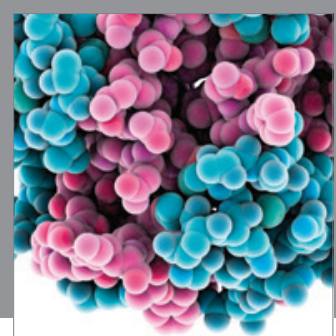

Journal of
Diabetes Research

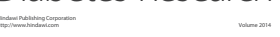

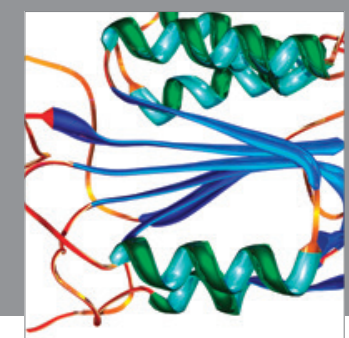

Disease Markers
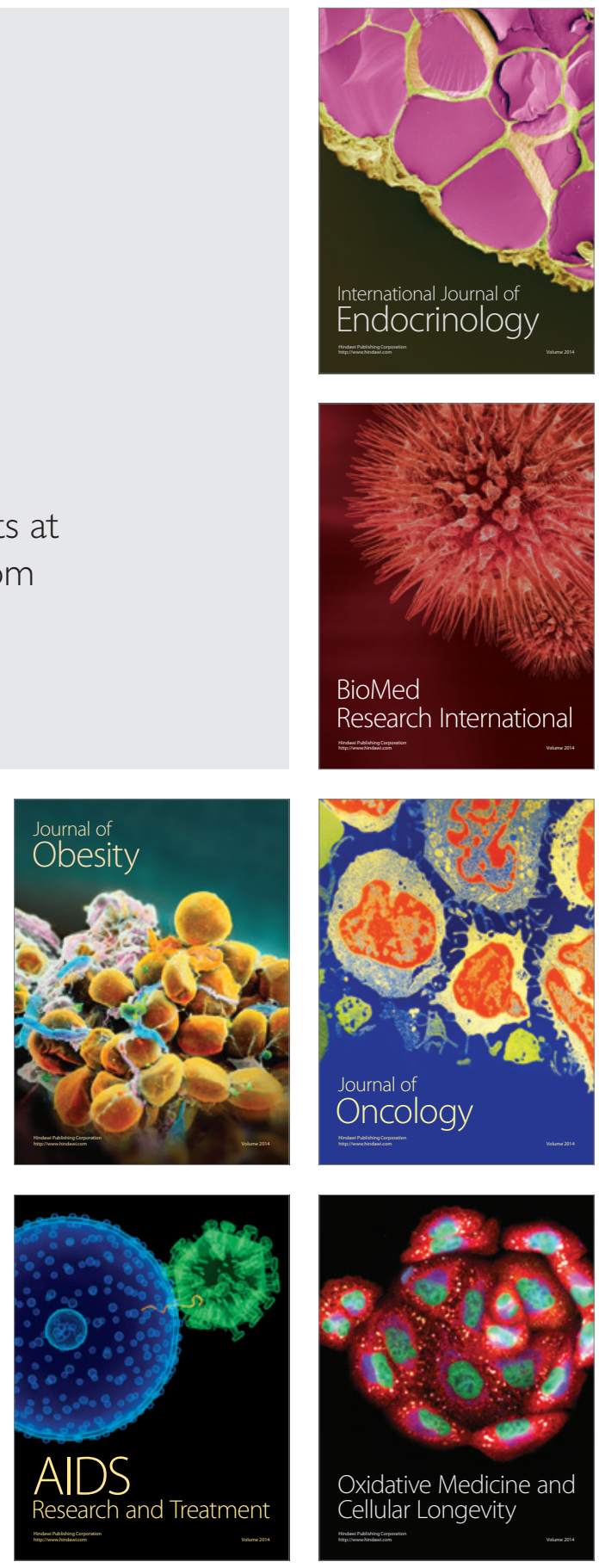